\title{
Parameter Tuning for Quad-rotor PID Control System Based on Optimal Latin Hypercube and PSO Algorithm
}

\author{
Muhao Chen ${ }^{1,}$, Chen Gong ${ }^{2, b}$, Xiaolong Bai ${ }^{3, \mathrm{c}}$ and Peilu Si ${ }^{4, \mathrm{~d}}$ \\ 1, 3, ${ }^{4}$ Room 208, School of Naval Architecture \& Ocean Engineering, Huazhong Universityof Science \\ and Technology, Wuhan, China \\ ${ }^{2}$ Room B214, School of Mechanical Science \& Engineering, Huazhong University of Scienceand \\ Technology, Wuhan, China \\ achenmuhao@hust.edu.cn, ${ }^{\mathrm{b}}$ gongchen_0812@hust.edu.cn, ${ }^{\mathrm{c}}$ baixiaolong@hust.edu.cn, ${ }^{\mathrm{d}}$ sipeilu@hus \\ t.edu.cn
}

Keywords: Opt LHD, PSO, Quad-rotor, PID

\begin{abstract}
Based on Optimal Latin Hypercube Design (Opt LHD) method and Particle Swarm Optimization (PSO) algorithm, this article presents a method for tuning PID control parameters of quad-rotor. Firstly, the range of 15 PID parameters are obtained by experience. Within the range of the PID parameters, Opt LHD method is applied to get few but evenly distributed test point groups, thus the adjustment work is reduced. Then, experiments for these PID test point groups are conducted and test results are used to establish response surface model (RSM). Finally, optimal PID parameter combinations are solved based on PSO Algorithm in this RSM. This method solves the following problems: 1 . large quantity of aimlessly repetitive tuning work; 2.getting global optimum PID parameters combinationswith personal experience. The final experiment results have been applied to quadrotor andthe validity of this method is proved.
\end{abstract}

\section{Introduction}

Quad-rotor has six degrees of freedom and attitude control is the core part of the entire control system $^{[1,2]}$. General algorithm models about attitude control are similar, and the mainwork is tuning the PID parameters. However, PID parameters tuning is mainly based on designers' experience now $^{[3]}$, which needs a large amount of repeating work, and it takes time to reach a stable state. To solve problems in PID parameters tuning, this paper presents a practical method combining optimal Latin hypercube and PSO algorithm.

\section{Quad-rotor Control System Modeling}

Quad-rotor has six degrees of freedom with only four independent drives, so it is an under-actuated and strong-coupling system.

Firstly, there are two basic coordinate systems: geodetic coordinate system (reference coordinate system) E (O-XYZ) and body coordinate system B (o-xyz). The relationship between the two coordinate systems are determined by three attitude angles, which are: roll angle $\varphi$, rotation about $\mathrm{x}$ axis in body coordinate system, and rotating to the right is positive; pitch angle $\theta$, rotation about y axis in body coordinate system, and rotating down is positive; yawangle $\psi$, rotation about $\mathrm{z}$ axis in body coordinate system, and rotation to the left is positive ${ }^{[4]}$.

Suppose the vehicle is rigid with the center of mass located at its geometric center, and three forces are applied to it: four lift forces $F_{i}, i=1,2, \cdots, 4$, which are proportional to rotational speed, gravity and air resistance. According to Newton's law and the relationship between body and geodetic coordinate systems, the acceleration can be calculated by Eq.1.

$$
\ddot{x_{n}}=\left(\sum_{i=1}^{4} F_{i}(\sin \varphi \sin \psi+\cos \varphi \sin \theta \cos \psi)-k_{1} \dot{x_{n}}\right) / m \text {. }
$$




$$
\begin{gathered}
\ddot{y_{n}}=\left(\sum_{i=1}^{4} F_{i}(-\sin \varphi \cos \psi+\cos \varphi \sin \theta \sin \psi)-k_{2} \dot{y_{n}}\right) / m . \\
\ddot{z}_{n}=\left(\sum_{i=1}^{4} F_{i}(\cos \varphi \cos \theta)-k_{3} \dot{z_{n}}\right) / m-g .
\end{gathered}
$$

$\mathrm{k}_{\mathrm{i}}, \mathrm{i}=1,2,3$ is air resistance coefficient in reference coordinate system, which can be neglected ${ }^{[5]}$.Similarly, rotational equations of quad-rotor in reference coordinate system can be obtainedbased on moment balance Eq.2:

$$
\ddot{\theta}=l\left(F_{4}-F_{2}-k_{4} \dot{\varphi}\right) / I_{x} . \quad \begin{gathered}
\ddot{\varphi}=l\left(F_{3}-F_{1}-k_{5} \dot{\theta}\right) / I_{y} .(2) \\
\ddot{\psi}=l\left(F_{1}-F_{2}+F_{3}-F_{4}-k_{6} \dot{\psi}\right) / I_{z} .
\end{gathered}
$$

$l$ is the distance from center of the rotor to center of the aircraft, and ki,i=4,5,6 is air resistance coefficient when three axes are rotating, which can be neglected too ${ }^{[5]}$.

Ignoring the air resistance and simplifying the relationship of lift force Eq.3.

$$
\left[\begin{array}{l}
u_{1} \\
u_{2} \\
u_{3} \\
u_{4}
\end{array}\right]=\left[\begin{array}{c}
F_{1}+F_{2}+F_{3}+F_{4} \\
F_{4}-F_{2} \\
F_{3}-F_{1} \\
F_{1}-F_{2}+F_{3}-F_{4}
\end{array}\right] .
$$

According to the relationship between translation and rotation, the quad-rotor control system model is Eq. ${ }^{[6]}$ :

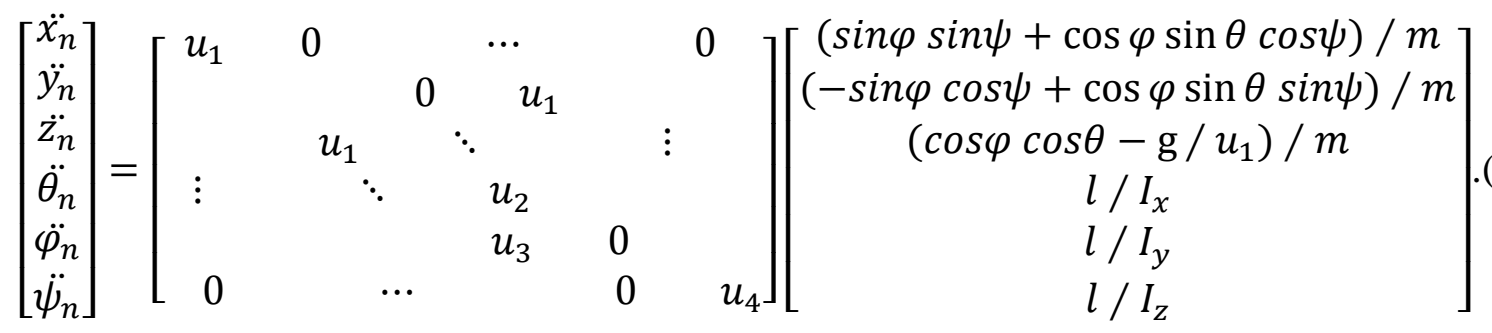

\section{PID Control}

All the results mentioned above lead to four control variablesu, dividing the complex nonlinear coupling model into four independent control channels ${ }^{[7]}$. It means the six freedom degrees of body can be controlled by $u_{1}, u_{2}, u_{3}, u_{4}$. Meanwhile, double-loopPID controller has better performance than a single loop. This paper uses the algorithm of double closed-loop control, andthe structure of the control unit is shown in Fig.1:

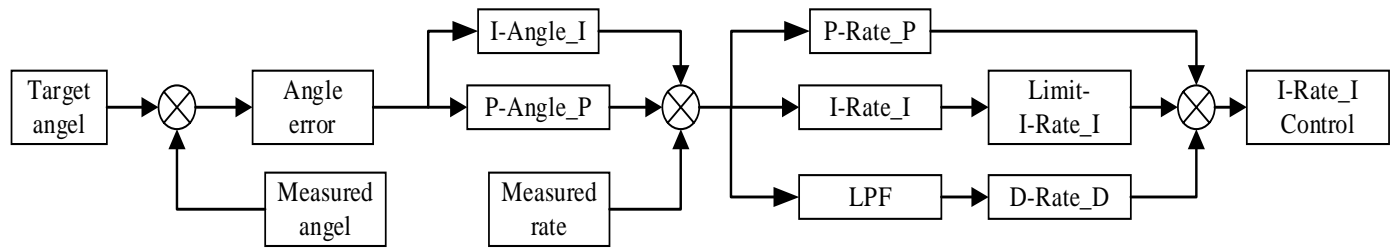

Fig. 1 The Structure of Double-loop PID Controller

Firstly, the angle error obtained by measuring current attitude angles and target attitude angles is multiplied by angle proportional coefficient $\mathrm{kp}_{1}$. Then, the angle error is integrated, and the result is 
multiplied by coefficient $\mathrm{ki}_{1}$. Add the two results above together and clip it as the target rate of angular velocity control unit ${ }^{[8]}$. The difference between target rate and gyroscope value is obtained and it is set as the input of the inner-loop controller.

\section{Optimum Latin Square and PSO to Optimize the Process of PID Tuning}

Problems remain in the traditional process of tuning the PID control parameters of the quad-rotor: 1) The amount ofexperiment points to be tested is large; 2) The direction of the tuning process is relatively ambiguous. Based on Optimum Latin Square and PSO, this paper solves the problems above by decreasing the number of the experiment samples and searching for the optimum solution more directionally. The concrete program flow chart is shown in Fig.2.

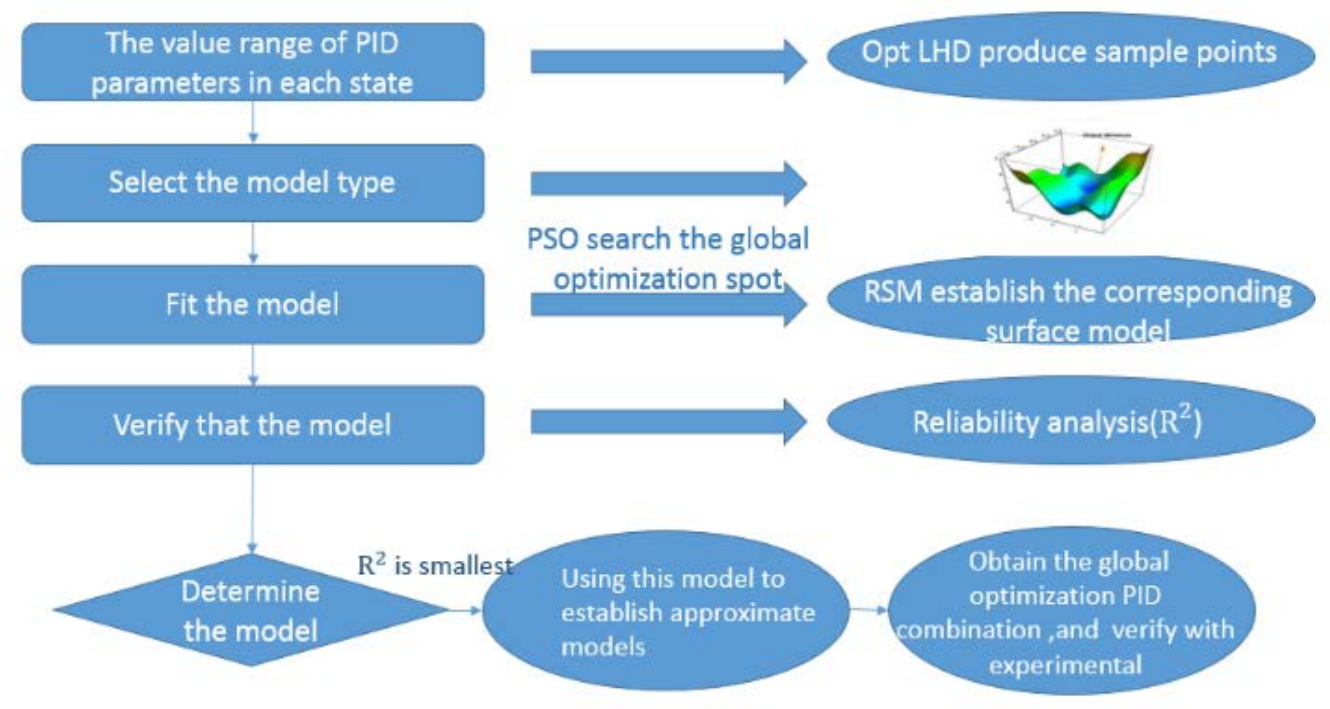

Fig. 2 Program Flow Chart

The multiple correlation coefficient $R^{2}$ is used as the index of error analysis to examine the accuracy of the established model. $R^{2}$ is defined in Eq.5:

$$
R^{2}=1-Q / S
$$

$\mathrm{Q}$ and $\mathrm{S}$ are residual sum of square and regression sum of square respectively. $R^{2}$ lies between 0 and 1 . The higher the value is, the higher accuracy and reliability the model has ${ }^{[9]}$.

\subsection{Orthogonal Experimental Design Based on Optimum Latin Square}

Advantages of Optimum Latin Square are as follows: 1) it is good at space filling. The number of sampling points needed is 81 in full factorial design while it is only 9 in Optimum Latin Square design; 2) it hashigh nonlinear response degree and uniform distribution of sampling points ${ }^{[10]}$. Compared with Orthogonal experiment, more combinations can be studied with the same number of sampling points. In an n-dimensional space, each one-dimensional coordinate interval $\left[x_{k}^{\min }, x_{k}^{\max }\right], k \in[1, n]$ is evenly divided into $\mathrm{m}$ intervals, and each small interval is denoted as $\left[x_{k}^{i-1}, x_{k}^{i}\right], i \in[1, m]$. Select m points randomly, and make sure each level of a single factor is studied only once,which will constituteann-dimensional space with $\mathrm{m}$ samples. This is am $x$ n Optimum Latin Square design, as shown in Fig.3(a), (b). 


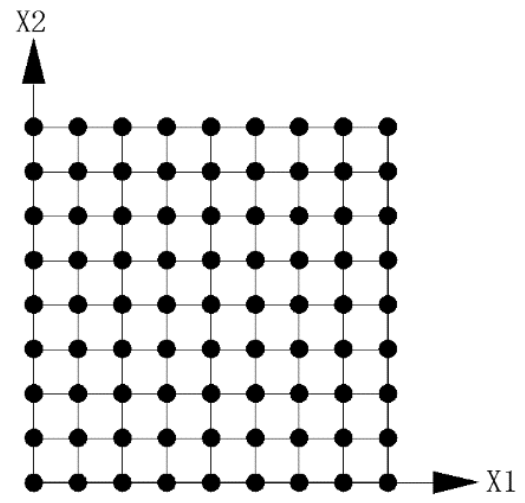

(a) Full Factorial design

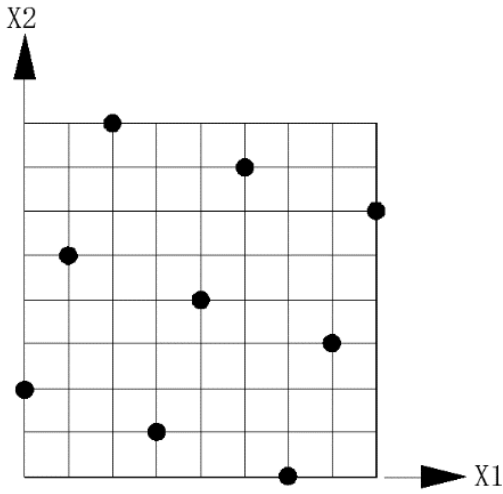

(b) Optimum Latin Square Design

Fig3: Full Factorial Design and Optimum Latin Square Design

The flight control system mentioned in this paper adopts the double-loop control structure: angle is the position loop (external loop,containing parameters $\mathrm{P}$ and I); rate is the speed loop (internal loop, containing parameters P, I and D), as shown in Table 1. The external loop of this cascade control is the master control part. The overall accuracy of this control system is determined by the external loop, and the internal loop plays a part of rough control.

Table 1 Value Ranges of PID Parameters

\begin{tabular}{|l|l|l|l|l|l|}
\hline Parameter & Angle_P & Angle_I & Rate_P & Rate_I & Rate_D \\
\hline Interval & $0.05 \sim 0.3$ & $0.001 \sim 0.01$ & $0.1 \sim 1.5$ & $0.001 \sim 0.01$ & $0.01 \sim 0.5$ \\
\hline Step & 0.001 & 0.0001 & 0.01 & 0.0001 & 0.001 \\
\hline
\end{tabular}

Roll, pitch and yaware the three states thatneed to be adjusted, and there are 5 parameters to adjust for each state. Applying the Optimum Latin Square to solve this problem will transform the process to an experiment design problem, and the interval range of the $5(\mathrm{~m}=5)$ dimension space is known. Then calculate $200 \quad(\mathrm{n}=200)$ distributed sample points combinations $\mathrm{X}\left\{A_{p i}, A_{I i}, R_{P i}, R_{I i}, R_{D i}\right\}, \mathrm{i} \in[1,200]$, and each combination contains 5 PID control parameters in the table 1.

Input the 200 combinations above to the aircraft one by one, then detect the deviation between the ideal response value and the measured one, denoting the deviation as $\Delta y_{i}$. We can obtainY $\left\{A_{p i}, A_{I i}, R_{P i}, R_{I i}, R_{D i}, \Delta y_{i}\right\}, \mathrm{i} \in[1,200]$. According to this result, the response surface model can be established, then take the optimal value (when the $\Delta y_{i}$ is minimum) as an initial particle value for PSO algorithm.

\subsection{Optimization of PID Parameters Based on PSO Algorithm}

To obtain the optimal combination of PID parameters for each channel, this article adopts Particle Swarm Optimization (PSO) algorithm. This algorithm has high efficiencyand goodconvergence to achieve optimization.

PSO is based on a group of particles. The solution for each optimization is to search for a birdin the space, which is called "particle". Every particle has its position. The $i_{\text {th }}$ particle is expressed as $X_{i}=\left(x_{i 1}, x_{i 2}, \cdots, x_{i D}\right)$ and the flying speed is expressed as $V_{i}=\left(v_{i 1}, v_{i 2}, \cdots, v_{i D}\right)$. Meanwhile, every particle has a fitness value decided by the objective function.

In every iteration of particle swarm, we need to find two extremum of particles. The updating formula of the speed and position of the particle is as Eq.6 and Eq.7:

$$
\begin{gathered}
v_{i d}=\omega \times v_{i d}+c_{1} \times r_{1} \times\left(p_{i d}-x_{i d}\right)+c_{2} \times r_{2} \times\left(p_{g d}-x_{i d}\right) . \\
x_{i d}=x_{i d}+v_{i d} .
\end{gathered}
$$

$v_{i d}$ is the $\mathrm{d}_{\mathrm{th}}$ dimensional speed of particle $\mathrm{i}$ in the $\mathrm{t}_{\mathrm{th}}$ iteration process. $x_{i d}$ is the current position of particle $i$ in the $t_{\text {th }}$ iteration. $\omega$ is inertia weight. Learning factors $c_{1}$ and $c_{2}$ are 
nonnegative constants. $r_{1}$ and $r_{2}$ are evenly distributed random numbers in $[0,1] . p_{i d}$ is the optimal value of current particles, and $p_{g d}$ is the global optimal value of the particle swarm ${ }^{[11]}$. The parameter setting in PSO Algorithm is shown in Table 2.

In this experiment, $\Delta y_{i}$ is calculated continuously. It works as afitness functionto update the position and speed of the particles. After 1000iterations, the parameter combination with minimum $\Delta y_{i}$ in this response surface can be obtained.

Table 2 Parameter Tuning of PSO Algorithm

\begin{tabular}{|c|c|c|c|c|c|c|}
\hline \multirow{2}{*}{$\begin{array}{c}\text { PSO } \\
\text { Coefficients }\end{array}$} & $\begin{array}{c}\text { Inertia } \\
\text { Weight }\end{array}$ & $\begin{array}{c}\text { Global } \\
\text { Increment } \\
\text { al }\end{array}$ & $\begin{array}{c}\text { Particle } \\
\text { Increment } \\
\text { al }\end{array}$ & $\begin{array}{c}\text { Number of } \\
\text { Particles }\end{array}$ & $\begin{array}{c}\text { Maximum } \\
\text { Iterations }\end{array}$ & $\begin{array}{c}\text { Maximum } \\
\text { Velocity }\end{array}$ \\
\hline 1 & 0.729 & 1.49 & 1.494 & & & \multirow{2}{*}{1000} \\
\hline 2 & 0.9 & 1.2 & 1.2 & 10 & 0.1 \\
\hline 3 & 0.72 & 2.04 & 1.15 & & \\
\hline
\end{tabular}

\section{Simulation Results}

The optimal solution combinations are solved using three groups of different parameters in PSO algorithm, as shown in Table 3. Combinations are tested in quadrotor and the minimum difference between actual angle value and ideal angle value indicates theoptimal result.Thecorresponding PID parameters combination is the optimal result for control system.

Table3 Actual Minimum Angle Values of PID Parameters

\begin{tabular}{|l|l|l|l|l|l|l|}
\hline PSO & Angle_P & Angle_I & Rate_P & Rate_I & Rate_D & $\begin{array}{l}\text { Difference } \\
\text { between Angles }\end{array}$ \\
\hline 1 & 0.0515 & 0.0991 & 1.4821 & 0.0022 & 0.0039 & 0.5 \\
\hline 2 & 0.0638 & 0.0088 & 1.372 & 0.0077 & 0.0073 & 0.8 \\
\hline 3 & 0.0519 & 0.0997 & 1.4997 & 0.0018 & 0.0043 & 0.5 \\
\hline
\end{tabular}

\section{Summary}

Simulation resultsand tests of quadrotor show that the Optimum Latin Square design method greatly reduces the test points, making the response surface model easier to build and improving the precision of PSO algorithm. Meanwhile, the optimization process are more automatic and directional, which can obtain better results.

\section{References}

[1] Yan Qingdong, Yu Tao, Zhu Lijun,Wei Wei:Efficiency control of hydrodynamic-mechanical transmission of engineering machinery. Journal of Jilin University Engineering and Technology Edition (2013).

[2] Wang Shichun:Optimal PID control for the four-rotor quadrotor. Journal of Hebei University of Science and Technology (2013).

[3] Ye Shuqiu, Zhan Lin: Quad-rotor Aircraft Attitude Control System Based on PID Control. Computer and Modernization (2015).

[4] Li Junfang, Zhang Buhan:Probabilistic Load Flow Based on Improved Latin Hypercube Sampling with Evolutionary Algorithm. Proceedings of the Chinese Society for Electrical Engineering (2011).

[5] Wu Chengfu, Liu Xiaoqi, Yuan Xu:Modeling and PID control for a quadrotor. Electronic Design Engineering (2012). 
[6] Zhang Weifeng, Che Yanbo, Liu Shengyang:Improved Latin Hypercube Sampling Method for Reliability Evaluation of Power Systems. Automation of Electric Power System (2015).

[7] Peng Anhua, Sun Xudong, Wang Zhiming:Parameter Tuning for PID Controllers Based on Orthogonal Experiment. Mechanical Science and Technology for Aerospace Engineering (2011).

[8] Lei Xiujuan, Fu Ali, Sun Jingjing:Performance analyzing and researching of improved PSO algorithm. Application Research of Computers (2010).

[9] Abhijit D, Frank L, Kamesh S. Backstepping:Approach for controlling a quadrotor using Lagrange form dynamics. Journal of Intelligent \& Robotic Systems (2009).

[10] Daewon L, Jin K H, Shankar S:Feedback linearization vs. adaptive sliding mode control for a quadrotor helicopter. International Journal of Control Automation and Systems (2009).

[11] Nicol C, Macnab C J B, Ramirez-Serrano A: Robust adaptive control of a quadrotor. Mecha-tronics(2011). 